\title{
SITUACIÓN DE RIESGO Y SALUD DE LOS ADOLESCENTES EN EL VALLE DE LOS RÍOS APURÍMAC, ENE Y MANTARO EN PERÚ
}

\author{
Fabiola Yeckting Vilela ${ }^{1, a}$
}

\begin{abstract}
RESUMEN
Se presenta el diagnóstico de las condiciones de vida de los adolescentes varones y mujeres en la zona del valle de los ríos Apurímac, Ene y Mantaro (VRAEM), quienes atraviesan situaciones de riesgo y vulnerabilidad social y sexual debido a los altos niveles de producción de la hoja de coca y el contexto del narcotráfico, que crean la ilusión del rápido ascenso social. A partir de ello, es necesario preguntarse ¿cuáles son las condiciones de vida y situaciones de riesgo de los adolescentes en el VRAEM? y ¿cuáles son las alternativas para una mejora de su situación desde el acceso a la educación y los programas de atención de su salud? Esta situación de riesgo es el resultado de las relaciones desiguales de género, violencia sexual, deserción escolar, embarazo adolescente, consumo de alcohol y drogas, así como de la falta de oportunidades como consecuencia de la participación en la economía de la coca y el narcotráfico entre los adolescentes en el VRAEM. Finalmente, se presentan los resultados de la atención de profesionales de los centros de salud para favorecer la consejería en salud sexual y reproductiva y el acceso a los servicios de salud del Programa Vida Adolescente, como oportunidad de vincular los contenidos del currículo de educación y las metas de cumplimiento de este sector, visibilizando la necesidad de un enfoque de interculturalidad e igualdad de género en el acceso a la salud y la educación de los adolescentes.
\end{abstract}

Palabras clave: Salud sexual; Adolescente; Tráfico de drogas, Embarazo de alto riesgo, Perú (fuente: DeCS BIREME).

\section{RISK AND HEALTH OF ADOLESCENTS IN THE VALLEY OF THE APURÍMAC, ENE AND MANTARO RIVERS IN PERU}

\begin{abstract}
The article presents the diagnosis of the living conditions of male and female adolescents in the area of the valley of the Apurimac, Ene and Mantaro rivers (in Spanish: VRAEM), who are exposed to situations of risk and social and sexual vulnerability and sexual due to the high levels of coca-leaf production and the context of the illegal drug trade which create the illusion of rapid social ascent. From this, it is necessary to ask: what are the living conditions and risk situations of adolescents in the VRAEM? And, what are the alternatives for an improvement of their situation from access to education and health care programs? This risk situation is the result of unequal gender relations, sexual violence, school dropout, teen pregnancy, alcohol and drug use, as well as of the lack of opportunities as a result of the participation in coca economy and illegal drug trade among adolescents in the VRAEM. Finally, we present the results of the attention of professionals of the health centers to promote counseling on sexual and reproductive health and access to health services of the Adolescent Life Program Life, as an opportunity to link the contents of the education curriculum and the compliance targets of this sector, highlighting the need of an approach of multiculturalism and gender equality in the access to health and education of adolescents.
\end{abstract}

Keys words: Sexual health; Adolescent; Drug trafficking, High-risk pregnancy; Peru (source: MeSH NLM).

\section{INTRODUCCIÓN}

El artículo presenta el diagnóstico de las condiciones de vida de los adolescentes varones y mujeres en la zona del valle de los ríos Apurímac, Ene y Mantaro (VRAEM), que atraviesan situaciones de desigualdad, riesgo y vulnerabilidad, debido a los altos niveles de producción de la hoja de coca y el contexto del narcotráfico, que crea la ilusión del rápido ascenso social, situación a partir de la cual es necesario preguntarse ¿cuáles son las condiciones de vida y situaciones de riesgo de los adolescentes en el VRAEM? y ¿cuál es la situación del acceso de los adolescentes a los programas de atención de su salud? Finalmente, en el artículo, los profesionales que participaron en el estudio analizan la realidad de atención de la salud sexual y su relación con los programas de incentivo de la educación.

\footnotetext{
Universidad Nacional Mayor de San Marcos, Lima, Perú

a Antropóloga, magíster en Antropología Andina

Recibido: 08/05/2017 Aprobado: 07/06/2017 En línea: 28/06/2017
}

Citar como: Yeckting Vilela F. Situación de riesgo y salud de los adolescentes en el valle de los ríos Apurímac, Ene y Mantaro en Perú. Rev Peru Med Exp Salud Publica. 2017;34(2):273-9. doi: 10.17843/rpmesp.2017.342.2867 
El presente artículo se basa en los resultados del "Estudio cualitativo sobre el incentivo monetario del programa JUNTOS sobre el Bono VRAEM", encargado por el Ministerio de Desarrollo e Inclusión Social de Perú. El estudio tuvo por objetivo general analizar las percepciones, usos, valoraciones y cambios generados por la entrega del incentivo monetario a los hogares, como estímulo a la educación secundaria de adolescentes entre los 14 a 19 años en el ámbito del VRAEM y evitar el abandono escolar por ingresar a la economía de la coca y el narcotráfico.

El análisis de la problemática de la situación de riesgo y vulnerabilidad de los adolescentes en el VRAEM, está basado en los resultados obtenidos en el trabajo de campo realizado en el marco de este estudio cualitativo de carácter etnográfico, el cual se realizó en cinco distritos (Kimbiri, Samugari, Sivia, Surcubamba y Colcabamba) ubicados en las zonas amazónicas y altoandina del VRAEM en los departamentos de Cusco, Ayacucho y Huancavelica. Las entrevistas y observaciones se llevaron a cabo en coordinación con las Unidades Territoriales del Programa JUNTOS y el MIDIS, entre los meses de noviembre de 2015 y febrero de 2016.

Para realizar la investigación, se consideró la diversidad geográfica y étnica, así como la inclusión de población de origen quechua y castellano hablante, migrante en los centros poblados, y la comunidad nativa asháninka de Sampantuari en Cusco. La muestra y preguntas de investigación fueron especificadas en los términos de referencia del estudio, que dieron como resultado una data de un total de 79 entrevistas de profundidad, 8 entrevistas grupales (56 personas, padres, madres y gestores locales) y 16 observaciones (actividades comunales, instituciones educativas y visitas a hogares con gestores locales).

\section{CONTEXTO DEL VRAEM}

El ámbito territorial del valle de los ríos Apurímac, Ene y Mantaro (VRAEM) comprende parte de los departamentos de Junín, Ayacucho, Cusco, Apurímac y Huancavelica. El VRAEM, según el INEI, para el año 2015 albergaba 616092 habitantes. El Decreto Supremo 040-2016-PCM, publicado el 10 de junio de 2016, reordena los ámbitos de intervención directa y de influencia en el VRAEM, que abarca un total de 65 distritos (29 distritos de intervención directa y 36 de influencia). Es una zona con gran diversidad ecológica y geográfica que cuenta con altitudes que van desde los 540 hasta los $3000 \mathrm{~m}$ de altitud. Recientemente, en el VRAEM se han creado cerca de 15 nuevos distritos, con el objetivo de mejorar la acción del estado en la zona.
En el año 2014, según datos de la Oficina de las Naciones Unidas contra la Droga y el Delito, en el VRAEM había 18845 hectáreas sembradas con coca, cultivadas con alta tecnología, con rendimientos promedio superiores a 3,5 TM/ha de hoja, obtenidas en 4 o 5 cosechas al año. La producción del VRAEM representa el $67,8 \%$ del total nacional, de una producción nacional que alcanza las 100840 toneladas métricas destinadas, en su mayoría, para el narcotráfico ${ }^{(1)}$.

Asimismo, en el ámbito del VRAEM se produce café y cacao para la exportación. Su volumen de producción es menor al de la economía de la coca, por lo que el narcotráfico sigue siendo uno de los principales problemas de esta zona. Según el Programa de Intervención VRAEM de la PCM: EI VRAEM constituye una zona de enorme interés estratégico para la continuidad de sus actividades ilícitas ${ }^{(2)}$. De esa manera, la presencia dominante del tráfico ilícito de drogas distorsiona la economía de la zona, por el blanqueo de los recursos financieros provenientes del narcotráfico (Figura 1).

\section{LA ADOLESCENCIA, ENFOQUE DE IGUALDAD DE GÉNERO E INTERCULTURALIDAD}

La adolescencia es la etapa de la vida de los seres humanos que se define de manera etaria entre los $12 \mathrm{y}$ los 17 a 22 años de edad. En ella se presentan cambios en las características sexuales, el aprestamiento, la formación de habilidades y capacidades, así como en los valores y percepciones. Ello condiciona la continuidad de pautas y formas de vida en el comportamiento cotidiano dentro del círculo familiar, a su vez, de conflicto social con el entorno y su realidad circundante. A partir de las transformaciones físicas, psíquicas y biológicas, así como de vínculos y estrategias de los adolescentes en los espacios locales, determinarán sus trayectorias de vida y sus proyectos hacia el futuro. Esta etapa es posible que se extienda hasta los 25 años y correspondería a un período de turbulencia y transición ${ }^{(3)}$.

El enfoque de igualdad de género identifica las características de las relaciones entre los hombres y las mujeres, según las condiciones de vida, diferencias y desigualdades. Este enfoque junto con el de interculturalidad, que visibiliza las diferencias culturales, debe ser intersectorial a la aplicación de políticas públicas, considerando la desigualdad étnica, las formas de violencia, racismo o discriminación. Por ello: La interculturalidad con enfoque de género parte del reclamo de los derechos culturales, de la multiculturalidad y del hecho de la diversidad cultural considerando la desigualdad de género ${ }^{(4)}$. Es importante la valoración identitaria en el VRAEM, diversa por las diferentes procedencias de su población de migrantes 


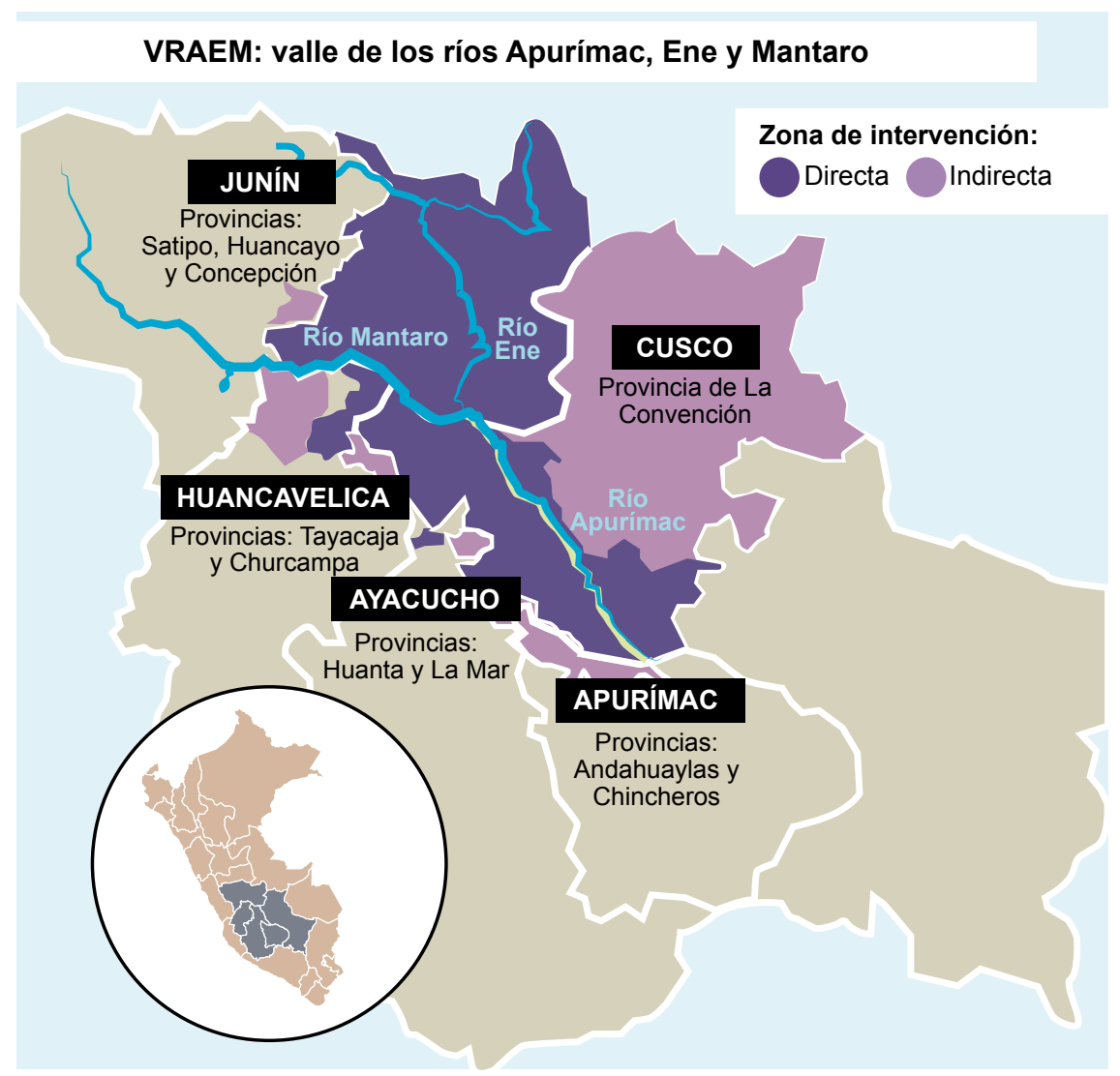

Figura1. Áreas de intervención directa y de influencia del valle de los ríos Apurímac, Ene y Mantaro (VRAEM) en el año 2016 (Fuente: http://rpp.pe/peru/narcotrafico/que-sucede-en-el-vraem-y-por-que-es-una-zona-tan-complicada-noticia-953452)

andinos y de poblaciones indígenas matsiguenga $\mathrm{y}$ asháninka. Debido a sus identidades culturales, las comunidades asháninkas plantean la articulación entre el saber indígena tradicional, la medicina de la posta y de las brigadas de salud ${ }^{(5)}$.

\section{RELACIONES DESIGUALES DE GÉNERO Y PROBLEMÁTICA ADOLESCENTE}

Las adolescentes tienen diferente acceso a oportunidades que configuran sus proyectos de vida. Ellas asumen las labores de apoyo en las tareas del hogar y de cuidado de hermanos menores y miembros de la familia. También existen madres solteras que cuidan y educan solas a sus hijos y, a su vez, quienes trabajan como vendedoras en puestos del mercado o negocios propios. En las familias monoparentales, son las adolescentes quienes se encargan de los hijos de madres, sobrinas, parientes, etc. Las mujeres se convierten a temprana edad en madres solteras o se ven obligadas a hacerse cargo de sus familias, debido a la ausencia o separación de su cónyuge por diversos motivos, que pueden ser: abandono, encarcelamiento o viudez. Sin embargo, cada vez son más importantes los proyectos de vida de las adolescentes, sus estudios y su futuro profesional.

Los niños y adolescentes varones, desde temprana edad, 7 o 8 años, frecuentemente van a las chacras a recoger la hoja de coca, se dedican al cultivo de esta planta y a su economía. Paralelamente, estudian y viven con sus madres y padres, apoyando en las labores agrícolas y del hogar hasta que entran a la edad de la adolescencia. Los padres la mayor parte del tiempo están ausentes, porque salen a trabajar a las chacras y las madres permanecen al cuidado de la familia. Es en este período cuando la mayoría de ellos deja el hogar, se independiza y busca trabajos temporales.

El inicio de la vida sexual de los adolescentes se orienta hacia la formación de una pareja, que puede determinar el embarazo y nacimiento de los hijos. La adolescente tomará decisiones según su percepción de los límites y posibilidades de sus condiciones de vida, las cuales estarán en función de valores, prácticas y conflictos en los que se manifestarán la carencia o soporte de sus redes familiares, sociales e identidades. Ello la diferencia 
del varón, porque en condiciones de precariedad y falta de oportunidades optarán por el vínculo sexual y de pareja como alternativa de cambio de vida: $A$ veces el problema es que quieran formar familia, buscando lo más fácil también, solucionan su problema económico de esa forma (Juan, director del Centro Educativo de Samugari). También optarán por trabajos temporales, como la atención en restaurantes y bares denominados "prostibares" en las zonas urbanas, que las expone a conductas de riesgo como la violencia sexual y la prostitución.

Al respecto, destacan tres situaciones: vulnerabilidad social, que acrecienta la vulnerabilidad sexual; machismo, que se expresa en situaciones de desigualdad, y violencia sexual, como comportamiento generalizado. También existe riesgo de violencia sexual en el trabajo doméstico y en el trabajo temporal, como el cultivo de la coca; así como una posición subordinada, debido a sus identidades étnicas y de clase social. Especialmente en algunas zonas del VRAEM se presenta como un hecho común generalizado ir semanalmente a "prostibares", destacando las situaciones de vulnerabilidad sexual en espacios de diversión de los adolescentes ${ }^{(6)}$. Las desigualdades de género estructuran los espacios sociales permitidos y prohibidos, y las normas sociales que incrementan la vulnerabilidad de los adolescentes frente a los riesgos sociales y de violencia sexual.

\section{DESERCIÓN ESCOLAR Y EMBARAZO ADOLESCENTE}

En los distritos como Samugari, Sivia e incluso Kimbiri, la economía de la coca afecta la educación. Así, los niños y jóvenes que se relacionan a diario con las posibilidades de 'emprendimiento', asociado al mercado directo e indirecto de la hoja de coca, acceden a equipos como smartphones, o ingresos que podrían hacerles creer que la educación no es importante. Los paradigmas de vida contrastan con lo que se enseña en un colegio público, porque se asocian con el "dinero fácil" y la "buena vida". Ello muestra que en la zona del VRAEM, asociada a la producción de hoja de coca y el narcotráfico, existe una "pobreza de tipo social", por lo que, contrariamente al flujo de dinero que hay en la región, los habitantes y profesores dicen "aquí no llega nadie", "aquí la vida no vale nada", y evidencia que "el estado no tiene nada que ver en la población", y que "no tiene injerencia" sobre ella, al estar sometida a otras dinámicas sociales no oficiales, informales ${ }^{(7)}$.

Según los directores de los centros educativos entrevistados, la deserción se da en la misma proporción entre hombres y mujeres. En menor medida los adolescentes desertan por dedicarse a trabajar y a apoyar en su familia. En tercero de secundaria se evidencia mayor deserción escolar. Similar situación encontramos en Samugari, según el comentario de un director: $A h i$ estaban señoritas del embarazo precoz. En este año como cuatro señoritas de tercero, una ya tiene su hijita, pero sigue estudiando, y me contaron que una chiquita de tercero está embarazada, pero sigue estudiando en cuarto. No tiene el apoyo, su papá está en la cárcel, su mamá falleció hace poco y está con su hermanita (Juan, director de Institución Educativa en el VRAEM).

En los Centros de Salud de Surcubambay Colcabamba en Huancavelica, zona de comunidades quechuahablantes, manifestaron que ha disminuido el embarazo adolescente, debido a las charlas de planificación familiar e ITS, tanto en la posta como en los colegios y la Academia "El Triunfador BECA 18". El estudio de Távara et al. ${ }^{(8)}$ muestra cómo el embarazo adolescente impacta en la salud física y estado de bienestar de las adolescentes. En el embarazo adolescente se presentan, además, una serie de complicaciones como síntomas, transformaciones, deficiencias e infecciones que deben ser atendidas por las instituciones, caso contrario, se convierten en pérdidas lamentables y hasta en la muerte de la gestante.

\section{CONSUMO DE ALCOHOL Y DROGAS}

Los adolescentes coinciden en que sus compañeros estudiantes que viven solos en la zona de valle comparten problemas como el aumento del alcoholismo, el pandillaje, el consumo de drogas y la vida nocturna en discotecas, tanto de los varones como de las mujeres, que puede derivar en la delincuencia y la prostitución. Eso sucede debido al fácil acceso a ingresos económicos que les brinda recoger coca, y dinero que gastan en una vida nocturna agitada. Por otra parte, los jóvenes que viven solos se enferman frecuentemente de fiebre tifoidea, paludismo, infecciones respiratorias, enfermedades diarreicas, entre otras. Asimismo, existen altos índices de desnutrición entre los niños y jóvenes en las comunidades, lo que conlleva a la falta de concentración en las horas de clase.

En la percepción de las madres y padres, las jóvenes por falta de apoyo familiar no logran encontrar un rumbo adecuado para sus proyectos de vida. Por lo que existen condiciones específicas de riesgo y de violencia de género en el VRAEM, que dificultan una mejor inserción educativa y laboral de las adolescentes. Los altos ingresos que genera el recojo de la hoja de coca y la pisa de las hojas de coca en las pozas de maceración como parte del proceso de elaboración de cocaína, expone a las adolescentes a la drogadicción y la prostitución.

Respecto a esta situación, y a cómo ello genera condiciones de riesgo para las adolescentes, una madre 
manifestó: Acá en VRAEM a veces falta de apoyo de madre, de padre. A veces las madres son irresponsables, no le apoyan a sus hijas, entonces ellas se meten a prostitución, a drogadicción. ¿Existe prostitución acá en Sivia? También hay, ¿y dónde ofrecen sus servicios? En todos los bares. Hay bastantes bares acá, ¿en dónde? En la entrada. A veces las mayorcitas se meten... venden licores desde los 16 y 17 años (María, madre de estudiante de Institución Educativa en el VRAEM). Por ello, considera que los bares son puerta de entrada a la prostitución, relaciones sexuales sin protección y a la drogadicción de jóvenes en edad escolar y de aquellas que han dejado el colegio.

En Samugari se encontró que las estudiantes coincidieron en que ya existe distribución de drogas en la institución educativa. El representante de CEDRO en Samugari explicó que existe un reciente consumo de marihuana entre los jóvenes en la institución educativa, aunque él atribuía la distribución a agentes externos al distrito. Al respecto el director del Centro Educativo manifestó /... ahora ya escucho consumo de marihuana, consumo de envoltorio de pasta básica a nivel de lo que viene a ser nacional. Aquí también está llegando, aqui también está llegando. Entonces, esos son los problemas, lo que está pasando (Juan, director de Institución Educativa en el VRAEM).

\section{PARTICIPACIÓN DE LOS ADOLESCENTES EN LA ECONOMÍA DE LA COCA Y EL NARCOTRÁFICO}

En Kimbiri, una familia que se dedica al cultivo de la hoja de coca realiza cosechas cuatro veces al año, mientras que la de café y cacao es solo una vez al año. Una madre de familia explicó que la coca es fundamental en la economía familiar, puesto que la cosecha de maíz da cada seis meses y la porción de tierra que siembran les da entre 3 a 4 sacos de 50 o 70 kilos cada uno. El cultivo de coca representa un $80 \%$ de su producción e ingresos. En el cultivo de cacao recibe S/ 25,00 por día como jornaleras. Las madres apoyan en la agricultura a sus esposos y venden sus productos en los mercados o en sus propios negocios.

Los habitantes del VRAEM prefieren producir la hoja de coca debido a que les genera el doble de ingresos económicos en comparación con otros cultivos, con ello garantizan los proyectos de vida para el futuro de sus hijos. Los jóvenes que estudian en el colegio van a recoger la hoja de coca los fines de semana, pero no se dedican al narcotráfico. Únicamente los miembros de la comunidad asháninka no producen cultivos de hoja de coca para vender, solo cacao y café, por lo que son considerados como pobres.
Otra característica de algunas de las zonas de Huancavelica es ser "de paso" para el narcotráfico, como camino para el traslado de mochilas con paquetes de cocaína. Esta modalidad de traslado la llevan a cabo adolescentes desde muy temprana edad y se convierte en una manera ilícita de conseguir altos ingresos en poco tiempo. Es un tema del cual no se habla y al que tratan de restar importancia. Según explica un docente en el VRAEM: Uno recomienda a los jóvenes que estudien, que trabajen, que más adelante les va a servir, y otros dicen: "No profe, yo me voy allá y al toque mi mochila y con eso gano más". O sea, quiere decir que lo han hecho, lo han visto, conocen ya...Se ha escuchado que pasan asi en grupos, hay ciertos tramos que se dispersan, así también, llevan por kilos. ¿Cuánto cargarán en una mochila?, a ver, calculando 7 kilos, 8 kilos. (Pablo, profesor de Institución Educativa en el VRAEM). Según la información de la zona, en estos recorridos de hasta dos semanas pueden recibir hasta 2000 dólares por un pase.

\section{IMPLICANCIAS EN SALUD}

El personal de los centros y puestos de salud de la zona intervenida, cumple labores de tiempo completo y reside en la zona, además está en permanente contacto con los pobladores, por ello tienen sus propias percepciones del comportamiento de los adolescentes y jóvenes, respecto a la salud en general y, en particular, de la sexual y reproductiva. Para conocer a este personal de salud se realizaron entrevistas, específicamente a los encargados del Programa Vida Adolescente, que se implementó hace alrededor de 10 años, y que tiene como objetivo la atención preventiva en salud de adolescentes, comprendiendo un rango de edad de 12 a 19 años ${ }^{(9)}$.

Según la especialista del Centro de Salud en el VRAEM, el acceso a la educación secundaria no está determinada por el género, es decir, los varones y mujeres tienen iguales oportunidades de acceso a la educación. Pero los trabajos específicos del sector Salud en la institución educativa se llevan a cabo de la siguiente manera: Los cursos y trabajos especificos en temas adolescentes se dan en la escuela, que son espacios públicos. De allí los adolescentes nos buscan en privado para hacernos preguntas, las mujercitas a nosotras las obstetras y los varoncitos a los choferes o enfermeros del centro de salud (María, promotora de centro de salud en el VRAEM).

El interés por la salud está presente en los adolescentes que solicitan más información, e identifican al personal del centro de salud por su género, y no precisamente por su especialidad: Son las propias alumnas que se acercan a los centros de salud a pedir consejería, además, se ofertan los métodos que tenemos, el 70\% 
de interés es de mujeres, $30 \%$ de varones. El inicio de la edad de preocupación por embarazos son los 15 años, principalmente de las mujeres. Hay antecedentes de embarazos con 13 años, que significa un riesgo enorme... (María jefa de Centro de Salud en el VRAEM). El personal de salud se preocupa por la actividad sexual de los jóvenes y adolescentes, sostiene que el interés por acceder a información y métodos de control de natalidad es principalmente de las mujeres.

El trabajo coordinado de las instituciones educativas y el sector salud no siempre van de la mano, en uno de los distritos visitados, se encuentra el siguiente testimonio, de la directora del centro de salud: Acá no hay apertura de los directores por estos temas. Hay prejuicios por parte de directores o profesores con algunos temas, como métodos anticonceptivos, ya que son vistos como incitadores a la práctica sexual, claro que esto ocurre solo en algunos colegios, en otros sí se puede trabajar" (Ana, directora de Centro de Salud en el VRAEM).

Según manifiesta la jefa de Obstetricia: La vida sexual en el distrito está empezando a temprana edad, a partir de los 13 años, según indagamos por propia voluntad, es decir, con sus parejas. Estos últimos años se han incrementado los embarazos a partir de los 13 y 14, y a esta edad hay complicaciones, riesgos, principalmente por la edad (Ana, directora del Centro de Salud de Colcabamba). La temprana edad de inicio de actividad sexual sin la adecuada orientación, podría traer como consecuencia inmediata, enfermedades de transmisión sexual, riesgo de embarazo y maternidad temprana.

En entrevista grupal en la comunidad asháninka de Sampantuari, mujeres adolescentes y jóvenes manifestaron que en los centros de salud y las postas no entienden la importancia del curandero y de la medicina tradicional que utilizan. Además, les prohíben ser acompañadas por sus hermanas o parientes cuando van a atenderse a los centros de salud, cuando van a dar a luz, y no las entienden porque el personal del servicio de salud desconoce sus costumbres, la medicina tradicional que utilizan y no comprenden el idioma asháninka.

Por los casos presentados, la posibilidad de coordinación y alianza entre el centro o puesto de salud y las instituciones educativas, determinará la orientación de la atención de la salud de los adolescentes. Si bien es una demanda conocer, principalmente, los métodos para la prevención de enfermedades de transmisión sexual, del embarazo adolescente y de control de natalidad, ya que están en edades de experimentación sexual, precisan de educación sexual y consejería sobre sus conductas de riesgo, vulnerabilidad y necesidad de oportunidades. De acuerdo a los resultados obtenidos, las instituciones educativas son el espacio adecuado para abordar estos temas, aunado al acompañamiento de acceso a la información y consejería del centro de salud en temas de derechos sexuales y reproductivos, siguiendo los principios de los enfoques de interculturalidad, adecuación cultural y de igualdad de género.

En la actualidad, no existe una articulación efectiva del sector Salud con el sector Educación para abordar una problemática en consejería en salud y educación sexual integral para los adolescentes de las localidades y comunidades en el VRAEM. Actualmente el Programa Vida Adolescente al que se afilian de manera voluntaria las familias usuarias de los servicios de salud, no se coordina con los centros educativos, salvo algunas excepciones, cuando podrían estar vinculados de manera directa desde las instancias del sector educación, e incorporando en el currículo educativo los temas de prevención de riesgos de la salud de los adolescentes. En el caso de los programas sociales, como el Programa JUNTOS, ello generaría condicionamientos de seguimiento del acceso a los programas de orientación, consejería, atención y prevención de enfermedades de los adolescentes.

En lo que respecta al estudio, los resultados muestran que en el VRAEM los adolescentes y jóvenes no reciben consejería y acompañamiento para prevenir las conductas de riesgo, violencia, desigualdad de género, discriminación y consumo de drogas, vinculados a la participación en la economía de la coca y el narcotráfico. En el caso del Bono VRAEM del Programa JUNTOS, el objetivo es prevenir la deserción escolar y asegurar la culminación de los estudios. Sin embargo, los hijos de las familias usuarias acceden al Bono, pero este no disminuye su participación en la economía de la coca, lo que hace que el primero se convierta en un ingreso adicional para proveer los materiales para la educación. Sin embargo, tampoco previene la vulnerabilidad sexual y social de los adolescentes, por lo que es importante promover programas integrales con otros sectores como Salud, además de Educación, así como proyectos productivos y de empleo, a nivel interinstitucional, y en las diferentes instancias del gobierno nacional, regional y local, para ampliar las posibilidades y mejorar el rumbo de sus proyectos de vida en la zona.

Agradecimientos: la autora agradece la participación en el estudio en el que se basa el artículo que también dirigió a los investigadores Dante Villafuerte, Renato Cáceres, Katheryn Cajavilca y Keithsy Inciso. También a Chaska Velarde y Elmer Guerrero del Ministerio de Desarrollo e Inclusión Social, quienes plantearon los objetivos del estudio y realizaron recomendaciones.

Conflictos de interés: los autores no presentan conflictos de interés. 


\section{REFERENCIAS BIBLIOGRÁFICAS}

1 Oficina de las Naciones Unidas contra la Droga y el Delito. Monitoreo de Cultivos de Coca en el Perú, 2014. Lima: Biblioteca Nacional del Perú; 2015. [Citado el 20 de abril del 2017]. Disponible en: https://www.unodc. org/documents/crop-monitoring/ Peru/Peru_Informe_monitoreo_ coca_2014_web.pdf

2 Díaz F. Los múltiples Senderos Luminosos en el actual Perú. En: VII Congreso del Instituto de Relaciones Internacionales de la Universidad de La Plata: 26, 27 y 28 de noviembre de 2014. La Plata, Argentina; 2014.

3 Feixa C. Antropología de las edades. En: Prat J, Martínez A. Ensayos de antropología cultural. Homenaje a Claudio Esteva-Fabregat. Barcelona: Editorial Ariel; 1996.

4 Rodríguez E, Iturmendi Vicente A. Igualdad de Género e Interculturalidad: enfoques y estrategias para avanzar en el debate [internet]. Programa de las Naciones Unidas para el Desarrollo; 2013. [Citado el 5 de marzo del 2017].
Disponible en: http://iknowpolitics. org/sites/default/files/atando_cabos_ may30.pdf

5 Central Asháninka del Río EneCARE. Kametsa Asaike. El vivir bien de los asháninkas del Río Ene. Agenda política de la CARE; 2011. [Citado el 10 de marzo del 2017]. Disponible en: http://www.proyecto-cbc.org.pe/ admin/recursos/publicaciones/18328OD-09.pdf

6 Yon Leau C. Teorías de cambio y buenas prácticas en salud sexual y reproductiva de los adolescentes: una relectura. Apuntes. 2015;42(76):9-36.

7 Avila D. ¿A qué se enfrenta diariamente un docente en el VRAEM? Un antropólogo te lo cuenta [Internet]. La mula.pe. 2017. [Citado el 25 de marzo del 2017]. Disponible en: https:// redaccion.lamula.pe/2015/11/11/aque-se-enfrenta-diariamente-undocente-en-el-vraem-un-antropologote-lo-cuenta/danielavila/
8 Távara L, Ordenique L, Sacsa $\mathrm{D}$, Oña J, Galecio Y, Garcia C, et al. Impacto del Embarazo en la salud adolescente (Perú). Lima: Centro de Promoción de los Derechos Sexuales y Reproductivos PROMSEX; 2015.

9 Ministerio de Salud. Norma Técnica para la Atención Integral de Salud en la etapa de Vida Adolescente. Lima: Dirección General de Salud de las personas. MINSA; 2005. [Citado el 6 de enero del 2017) Disponible en: http://bvs.minsa.gob.pe/local/ dgsp/131_NTAISADOL.pdf

Correspondencia: Fabiola Yeckting VilelaDirección: Calle Juan de Betanzos 163. San Miguel. Lima, Perú

Correo electrónico:fabiolayeckting@gmail.com

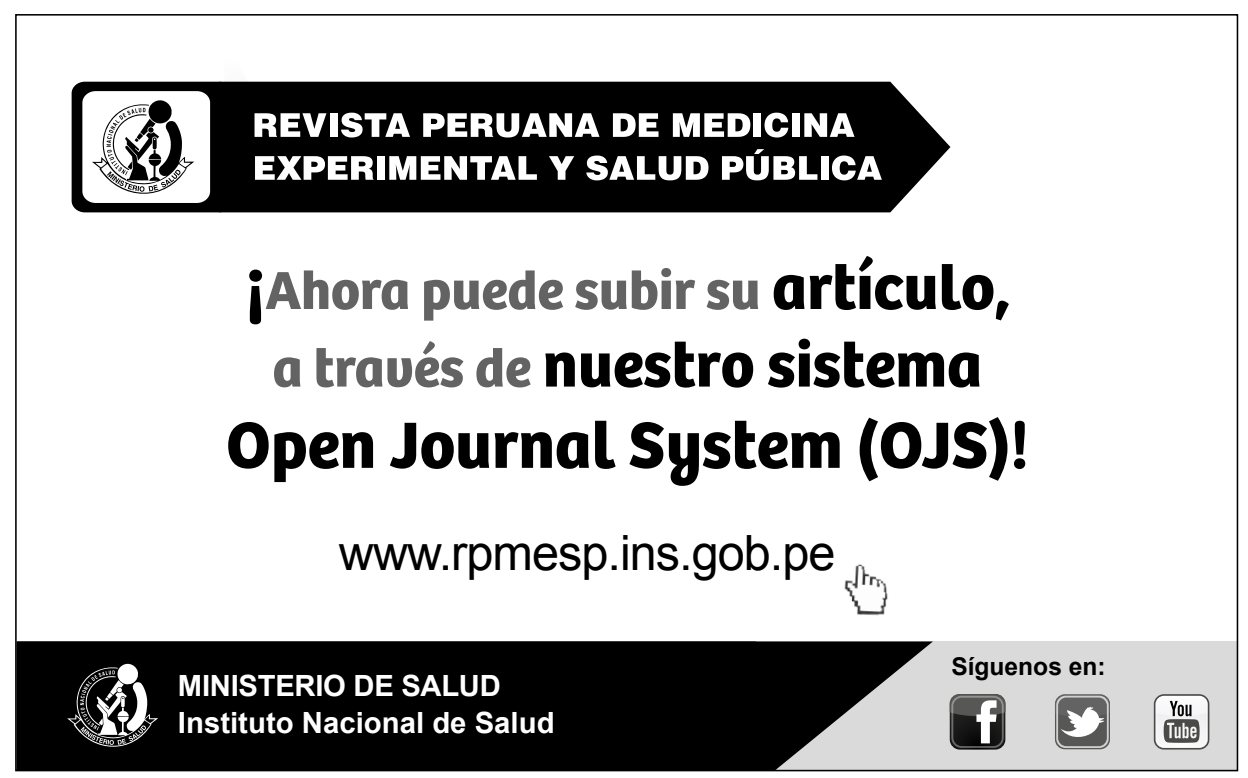

